\title{
Mode-Field-Diameter and the Coupling Loss between Inner and Outer Segment of Photoreceptors
}

\author{
Anhui Liang ${ }^{*}$, Leiting $\mathrm{Hu}^{1}$, Zhimin Liang2 \\ ${ }^{1}$ College of Opto-Electronic Engineering, Nanjing University of Posts and Telecommunications, Nanjing, China \\ ${ }^{2}$ Los Gatos High School, 20 High School Court, Los Gatos, USA \\ Email: "liangah@njupt.edu.cn
}

Received 2 March 2015; accepted 27 April 2015; published 30 April 2015

Copyright (C 2015 by authors and Scientific Research Publishing Inc.

This work is licensed under the Creative Commons Attribution International License (CC BY).

http://creativecommons.org/licenses/by/4.0/

(c) (i) Open Access

\begin{abstract}
The characteristics of optical waveguide of human photoreceptors play important roles in vision. The mode-field-diameter (MFD) is a very important parameter of a single-mode waveguide, and it is related to many important optical characteristics of a single-mode waveguide. Here we show that MFDs of outer segments of human foveal cones are close to the minimum values at their geometric diameter for outer segments of foveal cones. Small MFD of outer segment is important for eyes to have high spatial resolution and low interaction between neighboring cones. We propose that the ellipsoids of foveal cones act as spot size converters to reduce the coupling losses between myoids and outer segments.
\end{abstract}

\section{Keywords}

Photoreceptor, Mode-Field-Diameter, Spot Size Converter, Coupling

\section{Introduction}

The characteristics of optical waveguide of human photoreceptors play important roles in vision. Enoch first discovered that the outer segments of vertebrate photoreceptors are optical waveguides [1]. Snyder and Pask studied cone photoreceptors by using multimode theory [2] and they showed that an isolated individual foveal cone can produce the Stiles-Crawford-1 effect [3]. In 1998, the coauthor of this paper found that the outer segments of human foveal cones are single-mode optical waveguides, which can support the foveal cones to have the function of high resolution and fast response speed to light [4]. Vohnsen et al. treated the light coupling to

*Corresponding author.

How to cite this paper: Liang, A.H., Hu, L.T. and Liang, Z.M. (2015) Mode-Field-Diameter and the Coupling Loss between Inner and Outer Segment of Photoreceptors. Optics and Photonics Journal, 5, 151-160.

http://dx.doi.org/10.4236/opj.2015.54014 
and from photoreceptors as Gaussian beams, and applied LP modes (mainly fundamental mode) to analyze Stiles-Crawford effect [5] [6]. The coauthor of this paper found cones and rods have optimum structures, which can lead to saving much more energy than those of the photodetectors used in optical fiber communication systems. We proposed that the nucleus of these cells may have the function of current amplification [7]-[14]. We proposed to design the photodetectors and detector arrays (of optical fiber communication systems) by mimicking the structures of cones and rods, e.g., we can design single-photon detectors by mimicking rods which can detect single photon; we can design $40 \mathrm{Gbit} / \mathrm{s}$ and higher speed photodetectors with single-mode waveguide by mimicking the cones' outer segments which are single-mode waveguides. Anhui Liang is the first person to study single mode detectors, since he studied the single-mode detectors in human eyes as early as 1980s-1990s. Anhui Liang first reported the outer segments of foveal cones are single-mode fibers in 1998, and it was earlier than 2004 when the first commercial single-mode photodetectors were used in optical fiber communication systems. The $40 \mathrm{Gbit} / \mathrm{s}$ photodetectors are single-mode waveguides and with tapered shape, which were similar to cone's structure in human eyes [15]. The coauthors of this paper have found at least 8 golden ratio points in human vision and found that there are strong optical coupling effect between adjacent foveal cones, which is very important to make the opponent color theory and trichromatic theory of vision unified [6]-[9].

The mode-field-diameter (MFD) is a very important parameter of single-mode waveguides, and it is related to many important optical characteristics (e.g. coupling loss, angular misalignment loss, transverse offset loss, Rayleigh scattering loss, macro-bending loss, cross talk between two waveguides, effective area, the power percentage in the core and waveguide dispersion etc.) of single-mode waveguides [12] [13] [16] [17]. When the MFDs of the end faces of two fibers match with each other well, the coupling loss between them will be small. In this paper, we discuss the MFD of central foveal cones and rods.

In this paper, we first discuss the normalized frequency $\mathrm{V}$ of foveal cones and rods so that we can find out whether each part of photoreceptors works at single-mode or multimode; then we study the MFD of photoreceptors; finally we study the role of ellipsoids in foveal cones as spot size converters to reduce the coupling loss and we find the coupling loss is close to minimum at the golden ratio point.

\section{Methods}

The relative refractive index difference of a waveguide is defined as:

$$
\Delta=\frac{n_{1}^{2}-n_{2}^{2}}{2 n_{1}^{2}}
$$

where $n_{1}$ and $n_{2}$ are the refractive index of core and cladding respectively. The normalized frequency $V$ of circular optical fiber is defined as:

$$
V=\frac{\pi d n_{1} \sqrt{2 \Delta}}{\lambda}
$$

where $d$ and $\lambda$ are the diameter of core and wavelength respectively.

Anhui Liang et al proposed the Laplacian mode-field width for arbitrary noncircular optical waveguide [12] [13], in special case for circular optical fiber, the Laplacian mode-field width is reduce to the Petermann II MFD [16] [17]. The formula used to calculate Laplacian MFD is [18]:

$$
\frac{W_{L}}{d}=\frac{\sqrt{2}}{W} \frac{J_{1}(U)}{J_{0}(U)}
$$

where $W_{L}$ is the Laplacian MFD. $U$ and $W$ are the normalized radial phase constant and the normalized radial attenuation constant respectively. $J$ is the Bessel function.

For two optical waveguides working at fundamental mode, the coupling loss at the interface between them is given by [19]:

$$
L(\mathrm{~dB})=-10 \log \left\{\frac{\left[\iint E_{1}^{\prime}\left(x_{2}, y_{2}\right) E_{2}\left(x_{2}, y_{2}\right) \mathrm{d} x_{2} \mathrm{~d} y_{2}\right]^{2}}{\iint E_{1}^{2}\left(x_{1}, y_{1}\right) \mathrm{d} x_{1} \mathrm{~d} y_{1} \iint E_{2}^{2}\left(x_{2}, y_{2}\right) \mathrm{d} x_{2} \mathrm{~d} y_{2}}\right\}
$$


where $E_{1}$ and $E_{2}$ are the fundamental mode field distributions at the end faces of the two waveguides respectively, and $E_{1}^{\prime}$ is the mode field distribution after the fundamental mode field of waveguide 1 reaches the endface of waveguide 2 . The coupling loss between myoid and outer segment can be treated as the sum of two following parts approximately: the first part is the coupling loss between the interface of myoid and ellipsoid and the second part is the coupling loss between the interface of ellipsoid and outer segment. If there had not been ellipsoid, the coupling loss between myoid and outer segment would have happened only at the interface between the myoid and outer segment.

When the cores of two optical fibers are aligned well, the coupling loss at the interface between them can be calculated approximately by the following formula [20]:

$$
L(\mathrm{~dB})=20 \lg \left(\frac{W_{L 1}^{2}+W_{L 2}^{2}}{2 W_{L 1} W_{L 2}}\right)
$$

where $W_{L 1}$ and $W_{L 2}$ are the mode field diameters at the end faces of the two optical fiber respectively.

\section{Results}

\subsection{The Normalized Frequency $V$ of Photoreceptors}

People studied cones and rods by applying the optical model with three sections, which includes myoid, ellipsoid and outer segment [21]. In this paper, we first propose to study cones and rods by applying the optical model with five sections, which includes nucleus, outer fiber, myoid, ellipsoid and outer segment (Figure 1(a)).

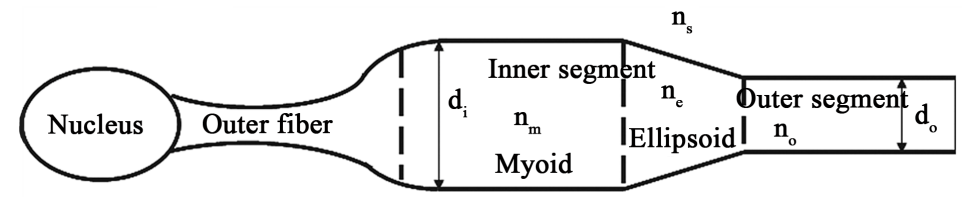

(a)

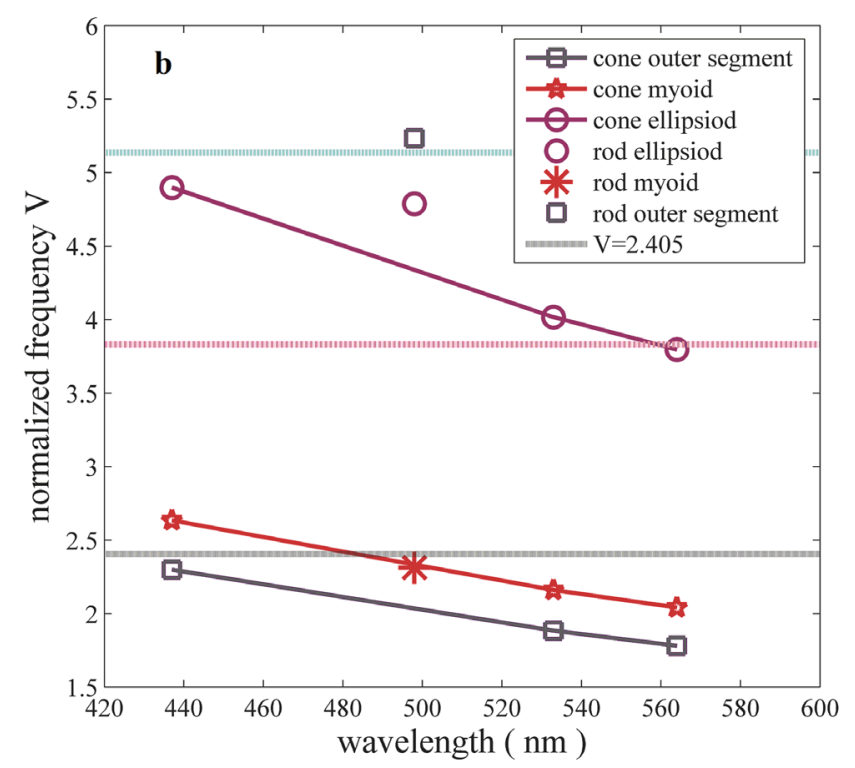

(b)

Figure 1. Five-section optical model of photoreceptors (a) and the normalized frequency $V$ of each part of photoreceptor cells (b). $d_{i}$ and $d_{o}$ are the diameters of inner and outer segment. $n_{m}$, $n_{e}, n_{o}$, and $n_{s}$ are refractive indexes of myoid, ellipsoid, outer segment and surrounding medium respectively. The pink dotted line represents $V=3.832$ and the blue one is $V=5.136$. When $V$ is between 2.405 and 3.832, there are total of two LP modes in optical fibers. When $V$ is between 3.832 and 5.136, there are total of four LP modes in optical fibers. When $V$ is between 5.136 and 5.52, there are total of five LP modes in optical fibers. 
In human eyes, there are rods and cones. The cones can contain different visual pigments and according to their absorption characteristics are called SWS, MWS and LWS cones (short, middle and long wave sensitive cones). The absorption maxima of SWS, MWS, LWS cones and rods are $437 \mathrm{~nm}, 533 \mathrm{~nm}, 564 \mathrm{~nm}$, and $498 \mathrm{~nm}$ respectively [22]. The inner and outer segment diameters of human foveal cones are about $2 \mu \mathrm{m}$ and $1 \mu \mathrm{m}$ in size respectively and the diameter of cones differs in going from the fovea to the periphery [23], while those of rods are both about $2 \mu \mathrm{m}$ [24]. To our knowledge, there are not the refractive indexes of human cones and rods available. But the values of refractive indexes of the same kind of cells in vertebrate species are close. In this paper, we take the average values of refractive index from different vertebrates species in Sidman's paper as human's refractive indexes, where the refractive indexes of myoid, ellipsoid, outer segment and surrounding medium are about 1.36, 1.39, 1.385 and 1.3476 for cones (and 1.36, 1.40, 1.41 and 1.3476 for rods) respectively [25].

When the normalized frequency $V$ of an optical waveguide is smaller than 2.405, which is the cut off normalized frequency of LP11 mode, it will work at single-mode. There is no mode interference in single-mode waveguides, therefore the light intensity in single-mode waveguides is more stable than that in multimode waveguides and the light, whose mode field distribution is like bell shape within the center at the fiber axis, is insensitive to environmental perturbation as well. As shown in Figure 1(b), the outer segments and myoids of foveal LWS and MWS cones are single-mode optical waveguides, which lead human cones to see objects very stable and with high spatial resolution and low noise in daylight. The outer segments of foveal SWS cones are single-mode optical waveguides as well, however the myoids of foveal SWS cones, whose normalized frequency is 2.635 which is slightly larger than 2.405 , work at multimode. Although the myoids of foveal SWS cones work at multimode, the main power is still contained in the fundamental mode instead of in high order modes.

The myoids of rods are single-mode waveguides, but the outer segments of rods are multimode optical waveguides. Because the light is launched from the myoids as the fundamental mode, the main power at the outer segments of rods is still contained in the fundamental mode. Because the rods and cones are responsible for scotopic and photopic vision respectively, one advantage for the outer segments of rods to have multimode optical waveguides structure is to improve sensitivity by gathering all of power in fundamental mode and high order modes.

The single-mode waveguide structure of foveal cones is good for their functions of high spatial resolution and high detection speed. Because the diameter of foveal cones' outer segments (about $1 \mu \mathrm{m}$ ) is smaller than that of rods (about $2 \mu \mathrm{m}$ ), the smaller diameter $d_{o}$ is helpful to reduce cones' capacitance and increase cones' response speed to light. In optical communication systems, $40 \mathrm{Gbit} / \mathrm{s}$ high speed photodetectors work at single-mode and are with tapered structure [15], and their structure is similar to the structure of foveal cones, which work at single-mode and are with tapered structure. In optical communication systems, 10Gbit/s and lower speed photodetectors work at multimode and are with straight structure, and their structure is similar to the structure of rods, which work at multimode and are with straight structure.

\subsection{Mode-Field-Diameter of Photoreceptors}

For arbitrary two dimensional single-mode waveguides (e.g. laser diodes, photo detectors etc.), there are several MFD definitions, but the Laplacian MFD, which was proposed by the coauthor of this paper and Fan, is the most important MFD definition [12] [13]. A photoreceptor's inner segment is hexagon [26], whose Laplacian MFD can be calculated from the MFD definition of arbitrary two dimensional optical waveguides. In this paper, we take the outer segment, ellipsoid and myoid as circular fibers for simplicity. In circular fibers, our Laplacian MFD definition for two dimensional waveguides will be simplified to the reference definition of MFD, which was proposed by Petermann [16] [17].

In this part, we mainly talk about MFDs of foveal cones, because the most part of rods work in multimode. Figure 2 shows the Laplacian MFD of outer segments of human foveal cones. As shown in Figure 2(b) and Figure 2(c), at cones' real geometrical diameter $(1 \mu \mathrm{m})$ and relative refractive index difference delta $=2.66 \%$ (calculated using the refractive index given by Sidman), the Laplacian MFDs of foveal MWS and LWS cones' outer segments are 1.34 and $1.42 \mu \mathrm{m}$ respectively, which are very close to the minimum values. It is worth to note that a small MFD is benefit to reduce the crosstalk between neighboring cone outer segments, where optical signals convert to electrical signals, therefore a small MFD is benefit to improve the spatial resolution. As shown in Figure 2(a), the Laplacian MFD $=1.14 \mu \mathrm{m}$ (at $d_{o}=1 \mu \mathrm{m}$ ) of SWS cones is close to the smallest 


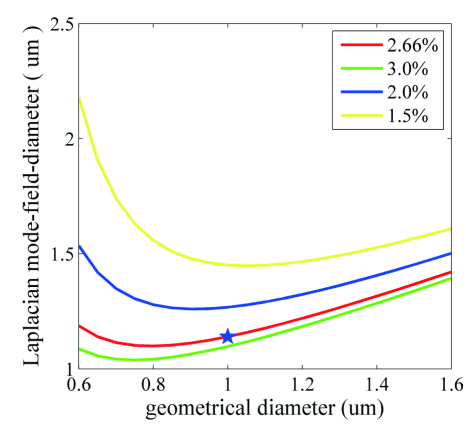

(a)

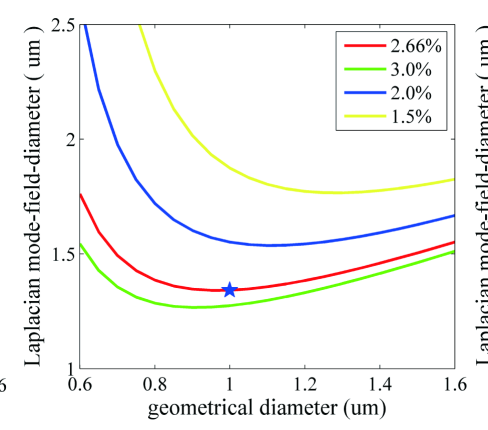

(b)

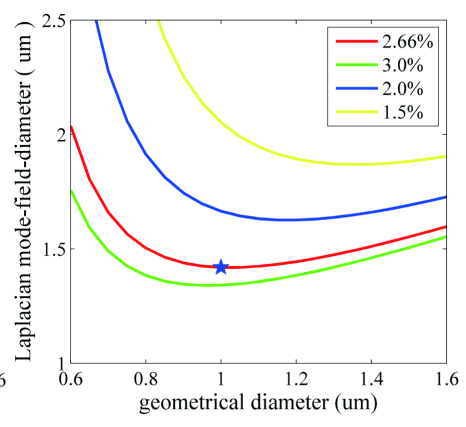

(c)

Figure 2. Laplacian mode-field-diameter Vs geometrical diameter of outer segment of human foveal cones. (a) Foveal SWS cone; (b) foveal MWS cone; (c) foveal LWS cone. The lines with different colors show the MFD at several relative refractive index differences of the outer segment. The red line in each figure shows the MFD at the relative refractive index difference of the outer segment calculated using the referenced refractive index given by Sidman. The blue star in each figure represents the point at the real geometrical diameter of the outer segment.

Laplacian MFD and its real geometrical diameter $1 \mu \mathrm{m}$. The Laplacian MFDs of foveal cones are sensitive to the relative refractive index differences.

Figures 3(a)-(c) show that Laplacian MFDs of photoreceptor's myoid are insensitive to the geometrical diameter variation around their real geometrical diameter (about $2 \mu \mathrm{m}$ ). Vohnsen et al. used the Gaussian MFD of $2.2 \mu \mathrm{m}$ of cones' inner segments in their model based on the Stiles-Crawford effect measurement and the Gaussian approximation [5] [6]. Our calculation results of MFD of cones' inner segments based on fiber parameters are close to their experimental results. The MFDs of myoids of foveal SWS, MWS and LWS cones are 2.05, 2.35 , and $2.46 \mu \mathrm{m}$ respectively. The diameter of outer fiber of foveal cones is about $1.6 \mu \mathrm{m}$ [27]. As shown in Figures 3(a)-(c), the MFDs at the geometrical diameter of $1.6 \mu \mathrm{m}$ and $2 \mu \mathrm{m}$ are nearly equal in foveal cones (which are 1.916, 2.303 and $2.455 \mu \mathrm{m}$ respectively for outer fibers of SWS, MWS and LWS cones), and this lead the coupling loss between myoids and outer fibers to be very small $(0.035,0.020$ and $0.022 \mathrm{~dB}$ respectively for outer fibers of SWS, MWS and LWS cones). We can found that there are strong coupling effects between inner segments of adjacent cones and outer fibers of adjacent cones, (see Ref. [7]), and these strong coupling are very important to unify the two important color theory: opponent color theory and tricolor theory.

Figure 4 shows the Laplacian MFDs of foveal cone myoid and outer segment change with the incident wavelength. The MFDs increase as the wavelength increases. This may be one of reasons that lead red objects appear larger than blue or green objects with same physical sizes. It is also interesting that the MFD of outer segment at long wavelength end is nearly equal to that of myoid at short wavelength end.

\subsection{Ellipsoids Act as Spot Size Converters to Reducing Coupling Loss}

The ellipsoid of inner segment of a cone is with tapered shape as shown in Figure 1(a). We propose that the ellipsoid of a cone may act as a spot size converter between myoid and outer segment to reduce coupling loss. The coupling loss between myoid and outer segment at each cone's most sensitive wavelength is calculated by Formula (4) and Formula (5) respectively in Figure 5 and we can see that the values are quite close. If the light had been launched from myoid to outer segment directly (i.e. without ellipsoid), the coupling losses between outer segment and myoid of foveal SWS, MWS and LWS cones would have been 1.39, 1.06, and $0.96 \mathrm{~dB}$ respectively (calculated with Formula (4)), which are quite large and will reduce the sensitivity and induce leaked light power significantly. As shown in Figures 5(a)-(c), if the foveal cones are with the ellipsoid in real situation, the minimum values of coupling losses between myoid and outer segment of foveal SWS, MWS and LWS cones are $0.21,0.33$ and $0.38 \mathrm{~dB}$ respectively (calculated with Formula (4)).

With the help of ellipsoids of foveal SWS, MWS and LWS cones, the coupling losses are reduced by 1.18, 0.73 and $0.58 \mathrm{~dB}$ respectively (at the optimum ellipsoid refractive index). This coupling loss reduction can help eyes to improve sensitivity and to reduce energy consumption; meanwhile it will help eyes to improve spatial resolution and to reduce noise because of very low leaked power to neighboring cones. The refractive index of ellipsoid is 1.39 according to Sidman's result, and our results show that the coupling losses of foveal SWS, 


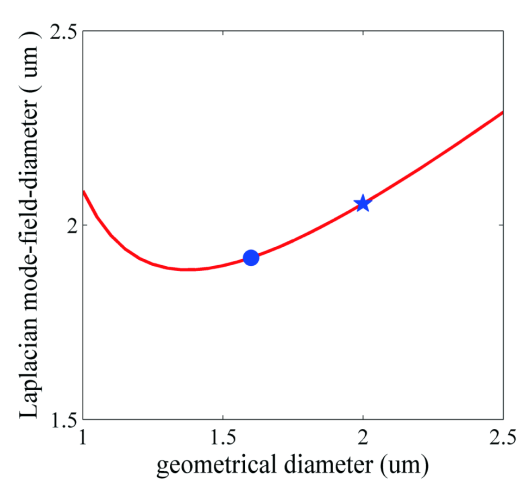

(a)

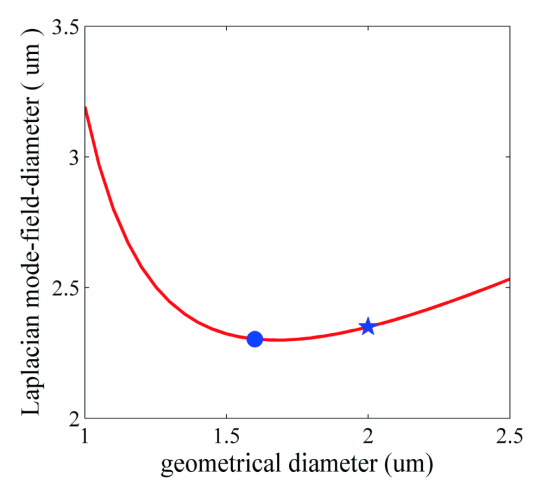

(b)

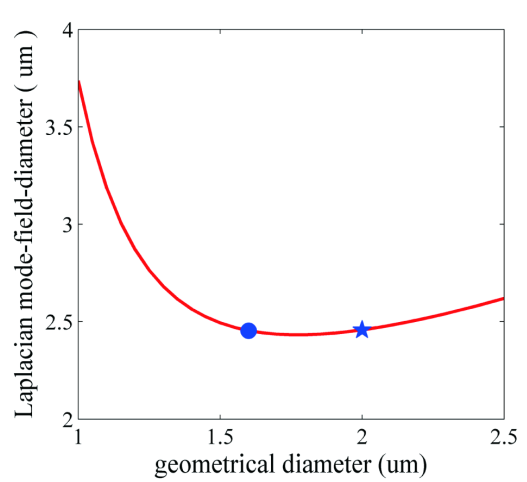

(c)

Figure 3. Laplacian mode-field-diameter of myoid and outer fiber vs geometrical diameter. (a) Foveal SWS cone; (b) Foveal MWS cone; (c) Foveal LWS cone. The blue star in each figure represents the point at the real geometrical diameter of myoid and the blue circle is the point at the real geometrical diameter of outer fiber.

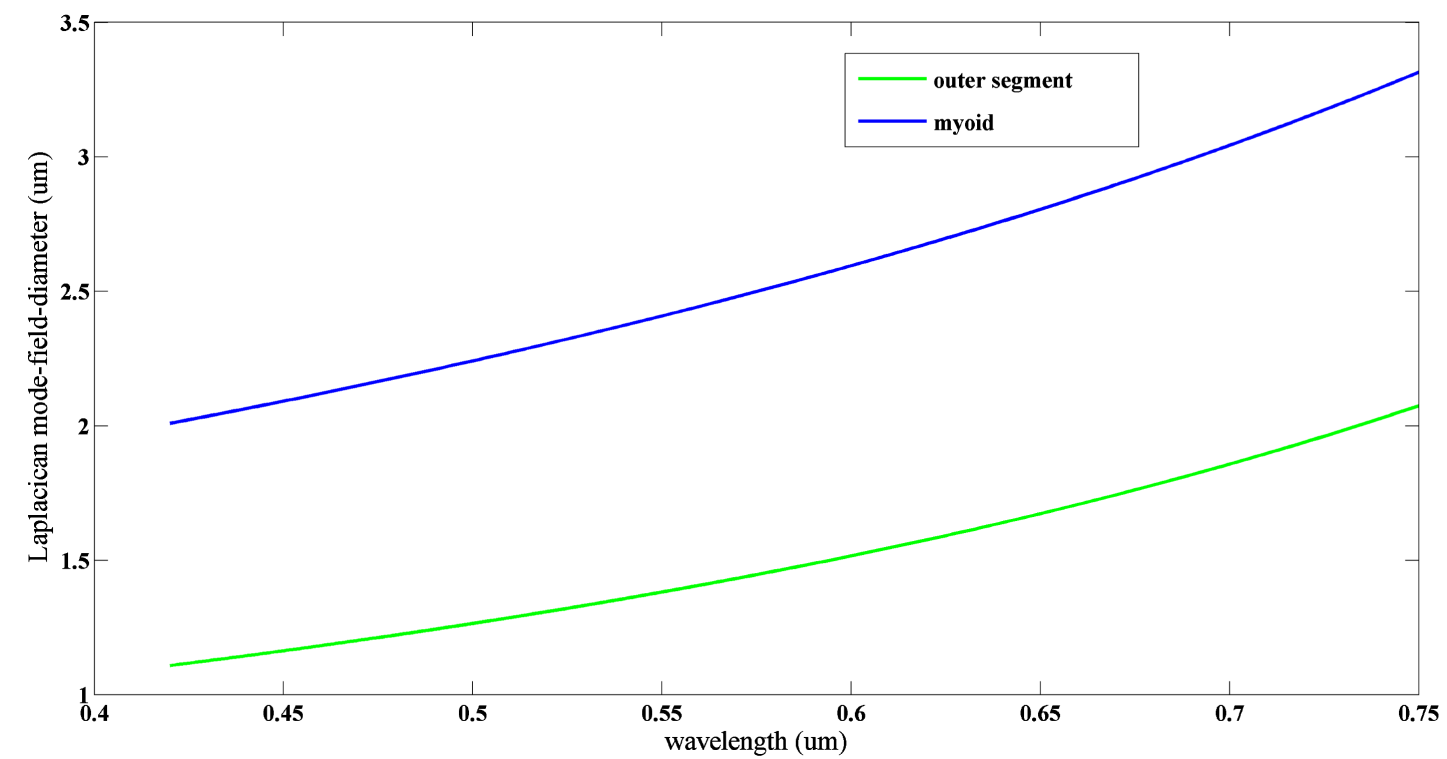

Figure 4. Laplacian mode-field-diameter of myoid and outer fiber Vs incident wavelength.

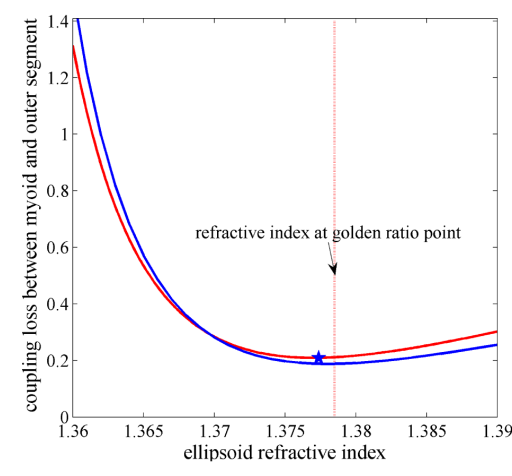

(a)

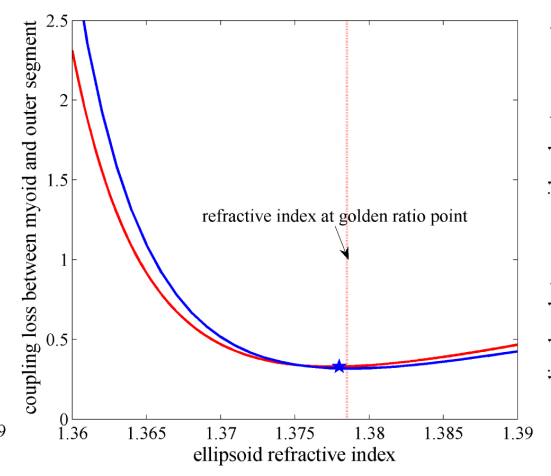

(b)

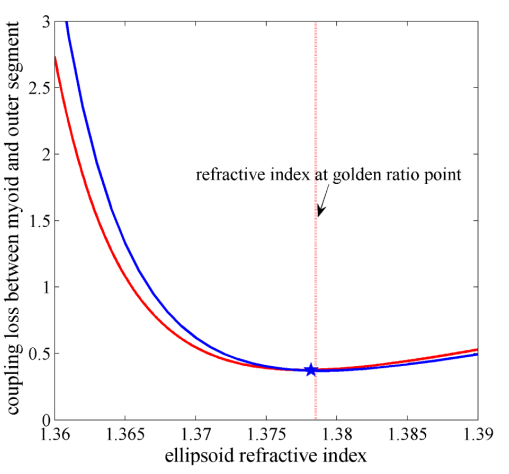

(c)

Figure 5. The coupling loss between myoid and outer segment vs ellipsoid's refractive index. (a) Foveal SWS cone; (b) Foveal MWS cone; (c) Foveal LWS cone. The red line in each figure is coupling loss calculated by Formula (4) and the blue one is that calculated by Formula (5). The blue star in each figure represents the point of the lowest coupling loss and the red dotted line is at the golden ratio refractive index between the refractive indexes of myoid and ellipsoid. 
MWS and LWS cones will be $0.30,0.46$ and $0.52 \mathrm{~dB}$ respectively at this refractive index (calculated with formula 4). With the helps of ellipsoid, the coupling losses of foveal SWS, MWS and LWS cones are reduced by 1.09, 0.60 and $0.44 \mathrm{~dB}$ respectively. At the minimum coupling loss points, the corresponding refractive indexes of ellipsoid of foveal SWS, MWS and LWS cones are 1.3774, 1.3780 and 1.3782 respectively, and they are quite close to the refractive index 1.3785 at the golden ratio point between myoid's refractive index $\left(n_{m}=1.36\right)$ and ellipsoid's refractive index $\left(n_{e}=1.39\right)$ (especially in MWS and LWS cones). As Figure 6 shows, nearly at all incident wavelengths, the coupling loss between foveal cone myoid and outer segment (calculated with formula 4) is at the lowest point when the refractive index is at the golden ration point value 1.3785.

We have calculated the coupling loss between foveal cone myoid and outer segment using formula 4 and 5 respectively, when the refractive index of ellipsoid is 1.3785. We can see from Figure 7 that the values of coupling loss calculated by Formulas (4) and (5) at the same wavelength are quite close when the MFDs are close to each other at adjacent end faces.

The coupling loss curves are quite flat in the right side region between the refractive index at golden ratio point and 1.39 in Figure 5. In this flat region, even if the ellipsoid's refractive index changes for some reasons (e.g., the amount of liquid in ellipsoid changes under some conditions etc.), the ellipsoid's function to reduce coupling loss still works well and is very stable. It is hard to measure the refractive index of photoreceptors in living condition very precisely, and it is possible that the real refractive index of foveal cone's ellipsoid in living condition may be smaller than that of Sidman's result 1.39 in some cases [28]. It is possible that the real refractive index of foveal cone's ellipsoid in living condition may be in the right side region between the refractive index at golden ratio point and 1.39, and even it is possible for the real refractive index to be at the golden ratio point to make the lowest coupling loss between myoid and outer segment, and this can save energy.

Figure 8 shows the function of foveal cones' ellipsoid as a spot size converter. Spot size converters, which can reduce coupling losses induced by MFD mismatch between two waveguides, have been widely used in single-mode fibers, laser diodes, photodetectors, modulators etc [29]-[33]. The MFD of a myoid is larger than that of the outer segment, and the MFD of ellipsoid reduces gradually from the interface between myoid and ellipsoid to the interface between ellipsoid and the outer segment. The ellipsoid of cones converts the large MFD to the small one in order to match the different MFDs of myoid and the outer segment. Therefore, we proposed that the ellipsoid of a cone acts as a spot size converter to reduce the coupling loss.

\section{Conclusions}

We first propose the five-segment optical model of human photoreceptors. We find MFDs of outer segments of

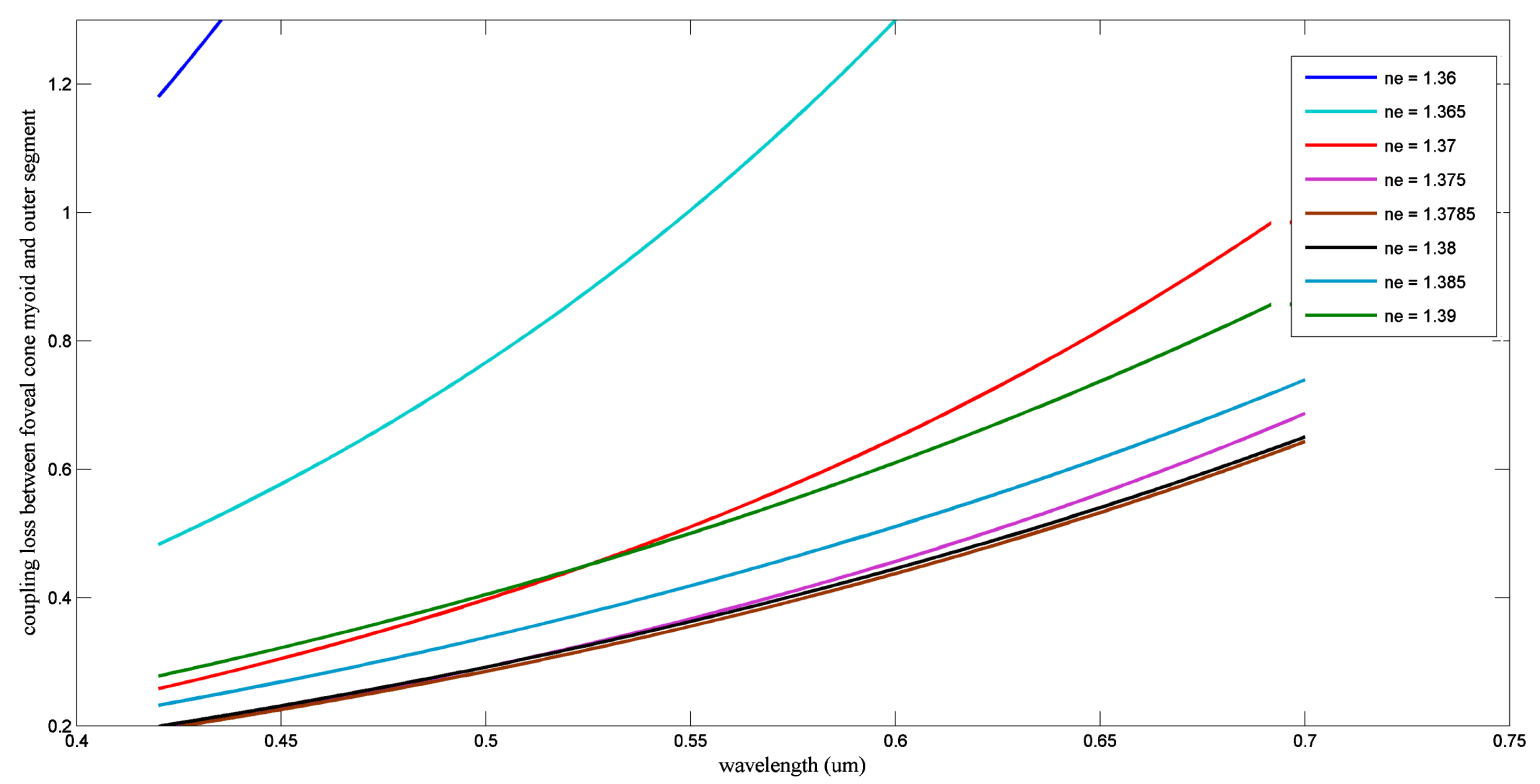

Figure 6. The coupling loss between myoid and outer segment of foveal cone cell at different refractive index of ellipsoid Vs incident wavelength. The refractive index of ellipsoid at the lowest line is 1.3785. 


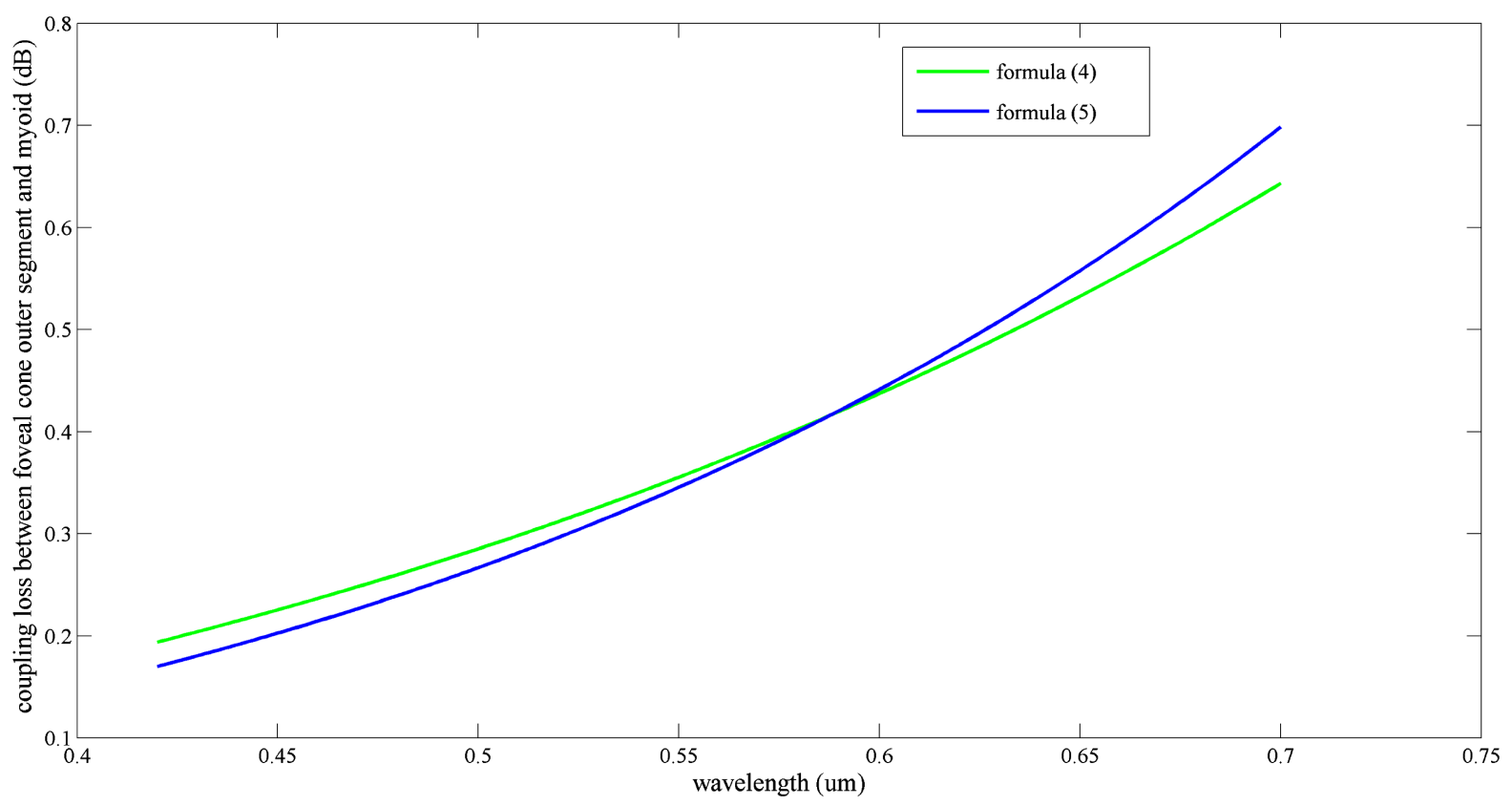

Figure 7. The coupling loss between myoid and outer segment of foveal cone cell when the refractive index of ellipsoid is 1.3785. The two lines are calculated with formula 4 and 5 respectively.

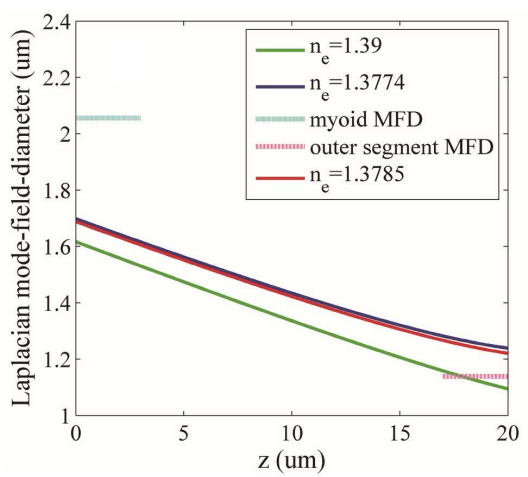

(a)

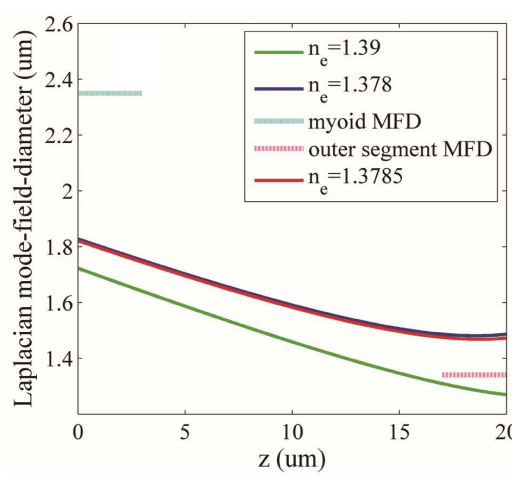

(b)

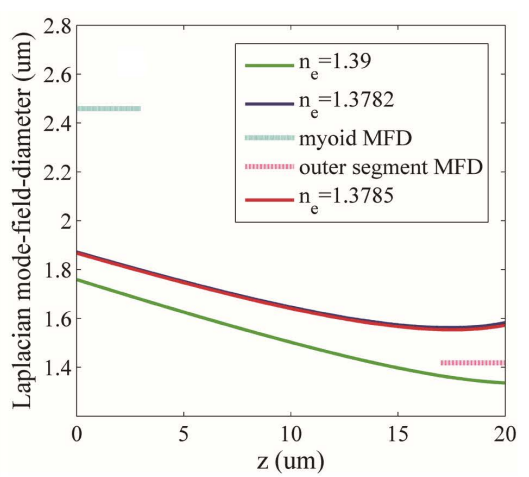

(c)

Figure 8. MFD matching effect of foveal cone ellipsoid by assuming that the length of ellipsoid is $20 \mu$ m. (a) Foveal SWS cone; (b) foveal MWS cone; (c) foveal LWS cone. $z$ is refered to the distance to the interface between myoid and ellipsoid. The meaning of the ' $n_{\mathrm{e}}$ 's are the refractive indexes appeared in the text above.

human foveal cones are close to the minimum values at their geometric diameter for outer segments of foveal cones. Small MFD of outer segment is important for eyes to have high spatial resolution and low interaction between outer segments of neighboring cones. We propose that the ellipsoids of foveal cones act as spot size converters to reduce the coupling losses between myoids and outer segments.

\section{Acknowledgements}

This work is supported by The National Natural Science Funds (81470661), Jiangsu Province Creative Talent and Startup Talent Program (0900613001), Jiangsu Province Creative Team Program, and NUPT Research Foundation for Advanced Talents (NY212001).

\section{References}

[1] Enoch, J.M. (1961) Visualization of Waveguide Modes in Retina Receptors. American Journal of Ophthalmology, 51, 1107-1118. http://dx.doi.org/10.1016/0002-9394(61)91800-1 
[2] Snyder, A.W. and Pask, C. (1973) The Stiles-Crawford Effect-Explanation and Consequences. Vision Research, 13, 1115-1137. http://dx.doi.org/10.1016/0042-6989(73)90148-X

[3] Stiles, W.S. and Crawford, B.H. (1933) The Luminous Efficiency of Rays Entering the Eye Pupil at Different Points. Proceedings of the Royal Society of London, 112, 428-450.

[4] Liang, A.H. (1998) Photoreceptors of Animals Are Quantum-Well Detectors. In: Proceedings of the 59th Autumn Meeting, The Japan Society of Applied Physics, paper 17p-ZA-1.

[5] Vohnsen, B., Iglesias, I. and Artal, P. (2005) Guided Light and Diffraction Model of Human-Eye Photoreceptors. Journal of the Optical Society of America A, 22, 2318-2328. http://dx.doi.org/10.1364/JOSAA.22.002318

[6] Vohnsen, B. (2007) Photoreceptor Waveguides and Effective Retinal Image Quality. Journal of the Optical Society of America A, 24, 597-607. http://dx.doi.org/10.1364/JOSAA.24.000597

[7] Liang, A.H. and Hu, L.T. (2014) Six-Segment Equivalent Circuit Models of Rods and Cones. Poster paper on Optical Society Vision Meeting, 10.

[8] Liang, A.H. and Hu, L.T. (2014) Strong Optical Coupling between Neighboring Cones on Human Retina. Poster paper on Optical Society Vision Meeting, 10.

[9] Liang, A.H. and Hu, L.T. (2014) Optical Fibers in Human Body and Optical Communication Bionics. WOCC, invited talk, Session OFDM and Visible Light Communications, Newark.

[10] Liang, A.H. and Hu, L.T. (2013) Novel Optical Waveguide Theory and Novel Electrical Circuit Theory of Photoreceptors in the Human Retina. In: Proceedings of PIERS, invited talk, Stockholm, Session 2P3.

[11] Liang, A.H. (2012) Optical Communication Bionics Brings New Ideas to Optical Devices and Modules in Future High Speed Networks. Proceedings of CIOE, Invited Talk, (Shenzhen, China), Session D01.

[12] Liang, A.H. (2011) High Speed Transponders, Systems and Optoelectronics-Bionics. Proceedings of Infostone Optical Fiber Communication Marketing and Technology, Invited Talk, (Wuhan, China).

[13] Liang, A.H. (1997) Transmission Characteristics Related to Laplacian Mode-Field Half-Width of Noncircular SingleMode Waveguides. Applied Optics, 36, 3793-3801. http://dx.doi.org/10.1364/AO.36.003793

[14] Liang, A.H. and Fan, C.C. (1998) Mode-Field Radius of Noncircular Field Single-Mode Fiber: New Definition and Application to Calculation of Splice Loss and Waveguide Dispersion. Electronics Letters, 24, 646-647. http://dx.doi.org/10.1049/el:19880438

[15] Honecker, J., Umbach, A., Trommer, D., Eckhardt, T. and Fischer, U.H.P. (2002) High-Speed Photo Diode Modules with up to $45 \mathrm{GHz}$ Modulation Bandwidth for Optical Communication Systems. Proceedings of OFC, Paper THGG 105.

[16] Petermann, K. (1983) Constraints for Fundamental-Mode Spot Size for Broadband Dispersion-Compensated Single Mode Fibers. Electronics Letters, 19, 712-714. http://dx.doi.org/10.1049/el:19830485

[17] Pask, C. (1984) Physical Interpretation of Petermann’s Strange Spot Size for Single-Mode Fibers. Electronics Letters, 20, 144-145. http://dx.doi.org/10.1049/el:19840097

[18] Hussey, C.D. and Martinez, F. (1985) Approximate Analytic Forms for the Propagation Characteristics of Single-Mode Optical Fibers. Electronics Letters, 21, 1103-1104. http://dx.doi.org/10.1049/el:19850783

[19] Fan, C.C. and Liang, A.H. (1990) Splice Loss between Different Gaussian-Elliptic-Field Single-Mode Fibers. Journal of Lightwave Technology, 8, 173-176. http://dx.doi.org/10.1109/50.47868

[20] Yu, S.X. (2002) Physics of Waveguide Theory. Press.njtu.edu, Beijing, 398-399.

[21] Michael, B., De Cusatis, C.M., Enoch, J.M., Lakshminarayanan, V., Li, G.F., MacDonald, C., et al. (2010) Biological Waveguides. In: Bass, M., Ed., Handbooks of Optics, 3rd Edition, Springer-Verlag, Berlin, 8.11-8.12.

[22] Georg, A.K. (2010) Industrial Color Physics. Springer, Berlin, 211-212.

[23] Yuodelis, C. and Hendrickson, A. (1986) A Qualitative and Quantitative Analysis of the Human Fovea during Development. Vision Research, 26, 847-855. http://dx.doi.org/10.1016/0042-6989(86)90143-4

[24] Hart, N.S. (2009) Retinal Photoreceptors. In: Binder, M.D., Hirokawa, N. and Windhorst, U., Eds., Encyclopedia of Neuroscience, Springer, Berlin, 3517-3522. http://dx.doi.org/10.1007/978-3-540-29678-2_5109

[25] Sidman, R.L. (1957) The Structure and Concentration of Solids in Photoreceptor Cells Studied by Refractometry and Interference Microscopy. The Journal of Cell Biology, 3, 15-30. http://dx.doi.org/10.1083/jcb.3.1.15

[26] Hoang, Q.V., Linsenmeier, R.A., Chung, C.K. and Curcio, C.A. (2002) Photoreceptor Inner Segments in Monkey and Human Retina: Mitochondrial Density, Optics, and Regional Variation. Visual Neuroscience, 19, 395-407. http://dx.doi.org/10.1017/S0952523802194028

[27] Hsu, A., Tsukamoto, Y., Smith, R.G. and Sterling, P. (1998) Functional Architecture of Primate Cone and Rod Axons. Vision Research, 38, 2539-2549. http://dx.doi.org/10.1016/S0042-6989(97)00370-2 
[28] Yamada, E. (1969) Some Structure Features of the Fovea Centralis in the Human Retina. Archives of Ophthalmology, 82, 151-159. http://dx.doi.org/10.1001/archopht.1969.00990020153002

[29] Soares, F.M., Karouta, F., Geluk, E.J., van Zantvoort, J.H.C., de Waardt, H. and Smit, M.K. (2004) Extremely LowLoss Vertically-Tapered Spot Size Converter in InP-Based Waveguide Structure. Proceedings of the 9th Annual Symposium of the IEEE/LEOS Benelux Chapter, Ghent, 2-3 December 2004, 127-130.

[30] Neumann, E.G. (1988) Single-Mode Fiber. Springer-Verlog, Berlin. http://dx.doi.org/10.1007/978-3-540-48173-7

[31] Marcuse, D. (1980) Radiation Losses of Step-Tapered Channel Waveguides. Applied Optics, 19, 3676-3681. http://dx.doi.org/10.1364/AO.19.003676

[32] Yoshimoto, N., Kawano, K., Hasumi, Y., Takeuchi, H., Kondo, S. and Noguchi, Y. (1994) InGaAlAs/InAlAs Multiple Quantum Well Phase Modulator Integrated with Spot Size Conversion Structure. IEEE Photonics Technology Letters, 6, 208-210. http://dx.doi.org/10.1109/68.275430

[33] Marcuse, D. (1970) Excitation of the Dominant Mode of a Round Fiber by a Gaussian Beam. Bell System Technical Journal, 49, 1695-1703. http://dx.doi.org/10.1002/j.1538-7305.1970.tb04285.x 